PROCEEDINGS OF THE

AMERICAN MATHEMATICAL SOCIETY

Volume 128, Number 2, Pages 521-525

S 0002-9939(99)05176-X

Article electronically published on June 24, 1999

\title{
LOMONOSOV'S THEOREM CANNOT BE EXTENDED TO CHAINS OF FOUR OPERATORS
}

\author{
VLADIMIR G. TROITSKY
}

(Communicated by David R. Larson)

\begin{abstract}
We show that the celebrated Lomonosov theorem cannot be improved by increasing the number of commuting operators. Specifically, we prove that if $T: \ell_{1} \rightarrow \ell_{1}$ is the operator without a non-trivial closed invariant subspace constructed by C. J. Read, then there are three operators $S_{1}, S_{2}$ and $K$ (non-multiples of the identity) such that $T$ commutes with $S_{1}, S_{1}$ commutes with $S_{2}, S_{2}$ commutes with $K$, and $K$ is compact. It is also shown that the commutant of $T$ contains only series of $T$.
\end{abstract}

All Banach spaces in this note are assumed to be infinite dimensional and separable; all operators are linear and bounded. We say that an operator is a non-scalar operator if it is not a multiple of the identity operator.

One of the major results in the history of the Invariant Subspace Problem was obtained by $\mathrm{V}$. Lomonosov in $[\mathrm{L}]$ who proved that if an operator $T$ on a Banach space commutes with another non-scalar operator $S$ and $S$ commutes with a nonzero compact operator $K$, then $T$ has an invariant subspace. Motivated by their study of the Invariant Subspace Problem for positive operators on Banach lattices, Y. A. Abramovich and C. D. Aliprantis have asked recently whether or not Lomonosov's theorem can be extended to chains of four or more operators. The purpose of this note is to answer this question in the negative. For our initial operator $T$ we will take an operator without an invariant subspace on $\ell_{1}$ coming from the famous construction of C. J. Read (see [R1]). Then we will produce three non-scalar operators $S_{1}, S_{2}$, and $K$ with $K$ compact (as a matter of fact $K$ has rank one) such that $T S_{1}=S_{1} T, S_{1} S_{2}=S_{2} S_{1}$, and $S_{2} K=K S_{2}$. This will be done in Section 1 .

After that, in Section 2, we will consider a related question of describing the commutant of the C. J. Read operator.

\section{A chain from C. J. Read's operator to a RANK-ONe operator}

We begin with reminding the reader of the construction in [R1] that will be central for us. As in [R1], we denote the standard unit vectors of $\ell_{1}$ by $\left(f_{i}\right)_{i=0}^{\infty}$. The symbol $F$ denotes the linear subspace of $\ell_{1}$, spanned by $f_{i}$ 's, and, thus, $F$ consists of eventually vanishing sequences.

Received by the editors March 31, 1998.

1991 Mathematics Subject Classification. Primary 47A15.

Key words and phrases. Invariant subspaces, commutant.

The author was supported in part by NSF Grant DMS 96-22454.

(c)1999 American Mathematical Society 
Let $\mathbf{d}=\left(a_{1}, b_{1}, a_{2}, b_{2}, \ldots\right)$ be a strictly increasing sequence of positive integers. Also let $a_{0}=1, v_{0}=0$, and $v_{n}=n\left(a_{n}+b_{n}\right)$ for $n \geqslant 1$. Then there is a unique sequence $\left(e_{i}\right)_{i=0}^{\infty} \subset F$ with the following properties:

0) $f_{0}=e_{0}$

A) if integers $r, n$, and $i$ satisfy $0<r \leqslant n, i \in\left[0, v_{n-r}\right]+r a_{n}$, we have $f_{i}=$ $a_{n-r}\left(e_{i}-e_{i-r a_{n}}\right)$;

B) if integers $r, n$, and $i$ satisfy $1 \leqslant r<n, i \in\left(r a_{n}+v_{n-r},(r+1) a_{n}\right)$ (respectively, $\left.1 \leqslant n, i \in\left(v_{n-1}, a_{n}\right)\right)$, then $f_{i}=2^{(h-i) / \sqrt{a_{n}}} e_{i}$, where $h=\left(r+\frac{1}{2}\right) a_{n}$ (respectively, $h=\frac{1}{2} a_{n}$ );

C) if integers $r, n$, and $i$ satisfy $1 \leqslant r \leqslant n, i \in\left[r\left(a_{n}+b_{n}\right), n a_{n}+r b_{n}\right]$, then $f_{i}=e_{i}-b_{n} e_{i-b_{n}}$

D) if integers $r, n$, and $i$ satisfy $0 \leqslant r<n, i \in\left(n a_{n}+r b_{n},(r+1)\left(a_{n}+b_{n}\right)\right)$, then $f_{i}=2^{(h-i) / \sqrt{b_{n}}} e_{i}$, where $h=\left(r+\frac{1}{2}\right) b_{n}$.

Indeed, since $f_{i}=\sum_{j=0}^{i} \lambda_{i j} e_{j}$ for each $i \geqslant 0$ and $\lambda_{i i}$ is always nonzero, this linear relation is invertible. Further,

$$
\operatorname{lin}\left\{e_{i}: i=1, \ldots, n\right\}=\operatorname{lin}\left\{f_{i}: i=1, \ldots, n\right\} \text { for every } n \geqslant 0 .
$$

In particular all $e_{i}$ are linearly independent and also span $F$. Then C. J. Read defines $T: F \rightarrow F$ to be the unique linear map such that $T e_{i}=e_{i+1}$, and in Lemma 5.1 he proves that $\left\|T f_{i}\right\| \leqslant 1$ for every $i \geqslant 0$ provided $\mathbf{d}$ increases sufficiently rapidly, i.e., satisfies several conditions of the form

$$
\begin{aligned}
& a_{n} \geqslant G\left(n, a_{0}, b_{0}, a_{1}, b_{1}, \ldots, a_{n-1}, b_{n-1}\right), \text { and } \\
& b_{n} \geqslant H\left(n, a_{0}, b_{0}, a_{1}, b_{1}, \ldots, a_{n-1}, b_{n-1}, a_{n}\right),
\end{aligned}
$$

where the $G$ and $H$ are some real-valued functions. It follows that $T$ can be extended to a bounded operator on $\ell_{1}$. Finally, C. J. Read proves that this extension, which is still denoted by $T$, has no invariant subspaces provided $\mathbf{d}$ increases sufficiently rapidly.

Throughout this section we will assume, without loss of generality, that all integers $a_{i}$ and $b_{i}$ are even. We are going to construct non-scalar operators $S_{1}, S_{2}$, and $K$ such that $K$ has rank one and commutes with $S_{2}, S_{2}$ commutes with $S_{1}$, and $S_{1}$ commutes with $T$. In fact, we take $S_{1}=T^{2}$, so that the equality $T S_{1}=S_{1} T$ is automatic. Define $S_{2}$ on $F$ via

$$
S_{2} e_{i}= \begin{cases}e_{i} & \text { if } i \text { is even } \\ 0 & \text { otherwise }\end{cases}
$$

We claim that

$$
S_{2} f_{i}= \begin{cases}f_{i} & \text { if } i \text { is even } \\ 0 & \text { otherwise }\end{cases}
$$

To prove this we consider all possible cases:

0) In this case $S_{2} f_{0}=S_{2} e_{0}=e_{0}=f_{0}$.

A) Since $a_{n}$ is even, then

$$
S_{2} f_{i}=a_{n-r}\left(S_{2} e_{i}-S_{2} e_{i-r a_{n}}\right)= \begin{cases}a_{n-r}\left(e_{i}-e_{i-r a_{n}}\right)=f_{i} & \text { if } i \text { is even } \\ 0 & \text { otherwise. }\end{cases}
$$

B) In this case

$$
S_{2} f_{i}=2^{(h-i) / \sqrt{a_{n}}} S_{2} e_{i}= \begin{cases}2^{(h-i) / \sqrt{a_{n}}} e_{i}=f_{i} & \text { if } i \text { is even; } \\ 0 & \text { otherwise }\end{cases}
$$


C) Since $b_{n}$ is even, we have

$$
S_{2} f_{i}=S_{2} e_{i}-b_{n} S_{2} e_{i-b_{n}}= \begin{cases}e_{i}-b_{n} e_{i-b_{n}}=f_{i} & \text { if } i \text { is even; } \\ 0 & \text { otherwise }\end{cases}
$$

D) Finally, in this case

$$
S_{2} f_{i}=2^{(h-i) / \sqrt{b_{n}}} S_{2} e_{i}= \begin{cases}2^{(h-i) / \sqrt{b_{n}}} e_{i}=f_{i} & \text { if } i \text { is even; } \\ 0 & \text { otherwise. }\end{cases}
$$

In particular, $S_{2}$ is bounded on $F$ and can be extended to $\ell_{1}$. For every $i \geqslant 0$ we have

$$
T^{2} S_{2} e_{i}= \begin{cases}T^{2} e_{i}=e_{i+2} & \text { if } i \text { is even } \\ 0 & \text { otherwise }\end{cases}
$$

On the other hand

$$
S_{2} T^{2} e_{i}=S_{2} e_{i+2}= \begin{cases}e_{i+2} & \text { if } i \text { is even } \\ 0 & \text { otherwise }\end{cases}
$$

so that $T^{2} S_{2} x=S_{2} T^{2} x$ for every $x \in F$. Since $F$ is dense in $\ell_{1}$, it follows that $T^{2}$ and $S_{2}$ commute on $\ell_{1}$.

Finally, define $K$ on $\ell_{1}$ via $K f_{0}=f_{0}$ and $K f_{i}=0$ for all $i>0$. Then $K$ is a bounded rank one operator on $\ell_{1}$, and $K$ commutes with $S_{2}$.

Note that if $m$ divides $a_{n}$ and $b_{n}$ for every $n$, then, similar to the previous construction, we could take for $S_{1}$ the operator $T^{m}$ instead of $T^{2}$. It follows from Lomonosov's theorem that $T^{m}$ has an invariant subspace (confer [R1, Lemma 6.4]).

In [R2] C. J. Read presents as a modification of his original example a quasinilpotent operator on $\ell_{1}$ without closed nontrivial invariant subspaces. The same argument as above provides a chain of four commuting operators connecting this operator to a compact operator.

\section{Commutants of C. J. Read's operators}

Let $\mathfrak{M}$ denote the collection of all real infinite matrices $A=\left(a_{i j}\right)_{i, j=0}^{\infty}$. Elements of $\mathfrak{M}$ can be added entrywise, the zero and identity matrices are defined in the natural way. Let $\mathcal{F}$ denote the subfamily of $\mathfrak{M}$ consisting of the matrices with a finite number of nonzero entries in every column and every row. For $A, B$, and $C$ in $\mathfrak{M}$ we say that $A B=C$ if for all $i, j \in \mathbb{N}$ we have $a_{i j}=\sum_{k=0}^{\infty} b_{i k} c_{k j}$ and the series converge absolutely. Though $A B$ may not exist in general, $A B$ exists if $A$ or $B$ belongs to $\mathcal{F}$.

We can also define the action of a matrix on a sequence: we say that $A x=y$ for $A \in \mathfrak{M}$ and $x, y \in \mathbb{R}^{\mathbb{N}}$ if $y_{i}=\sum_{j=0}^{\infty} a_{i j} x_{j}$ for every $i \geqslant 0$. Again, if $A \in \mathcal{F}$, then $A x$ is defined for every $x \in \mathbb{R}^{\mathbb{N}}$. Let $A^{(j)}$ denote the $j$-th column of $A \in \mathfrak{M}$; then $A^{(j)}=A f_{j}$. Finally, $(A)_{i j}$ or $a_{i j}$ will denote the $(i, j)$-th entry of $A \in \mathfrak{M}$.

The space $\mathcal{B}\left(\ell_{1}\right)$ of all (bounded) operators on $\ell_{1}$ can be naturally embedded in $\mathfrak{M}$ : if $R \in \mathcal{B}\left(\ell_{1}\right)$, then $r_{i j}=\left(R f_{j}\right)_{i}$. Obviously, the sum of operators in $\mathcal{B}\left(\ell_{1}\right)$ corresponds to the sum of matrices in $\mathfrak{M}$. Moreover, the action of $R$ on an element of $\ell_{1}$ is in accord with the definition of the action of a matrix on a sequence: if $x=\left(x_{1}, x_{2}, \ldots\right) \in \ell_{1}$ then $x=\sum_{j=0}^{\infty} x_{j} f_{j}$, so that $R x=\sum_{j=0}^{\infty} x_{j} R f_{j}$, and $(R x)_{i}=\sum_{j=0}^{\infty} x_{j}\left(R f_{j}\right)_{i}=\sum_{j=0}^{\infty} r_{i j} x_{j}$. Also, the product of two operators in $\mathcal{B}\left(\ell_{1}\right)$ corresponds to the product of two matrices: if $R, P \in \mathcal{B}\left(\ell_{1}\right)$, then $R P f_{j}=R P^{(j)}=$ $R \sum_{k=0}^{\infty} p_{k j} f_{k}=\sum_{k=0}^{\infty} p_{k j} R f_{k}$, so that $(R P)_{i j}=\left(R P f_{j}\right)_{i}=\sum_{k=0}^{\infty} p_{k j} r_{i k}$. Notice 
that two operators in $\mathcal{B}\left(\ell_{1}\right)$ commute if and only if they commute as matrices in $\mathfrak{M}$. Finally, the identity and zero operators correspond to the identity and zero matrices respectively.

Let $S$ be the right shift operator, i.e., $S f_{j}=f_{j+1}$. Given a formal power series $p(t)=\sum_{n=0}^{\infty} p_{n} t^{n}$, the matrix $p(S)=p_{0} I+p_{1} S+p_{2} S^{2}+\ldots$ belongs to $\mathfrak{M}$ and is of the form

$$
\left(\begin{array}{ccccc}
p_{0} & 0 & 0 & 0 & \ldots \\
p_{1} & p_{0} & 0 & 0 & \ldots \\
p_{2} & p_{1} & p_{0} & 0 & \ldots \\
p_{3} & p_{2} & p_{1} & p_{0} & \ldots \\
\vdots & \vdots & \vdots & \vdots & \ddots
\end{array}\right) .
$$

On the other hand, every matrix of this form is obviously a series of $S$.

Lemma 1. If $A \in \mathfrak{M}$ commutes with $S$, then $A=p(S)$ for some series $p$.

Proof. Since

$$
(A S)_{i j}=\sum_{k=0}^{\infty} a_{i k} s_{k j}=a_{i, j+1}
$$

and

$$
(S A)_{i j}=\sum_{k=0}^{\infty} s_{i k} a_{k j}= \begin{cases}a_{i-1, j} & \text { if } i \geqslant 1 \\ 0 & \text { if } i=0\end{cases}
$$

for every pair $(i, j)$, it follows that $A$ is of the form (1).

Consider $Q \in \mathfrak{M}$ such that $Q f_{j}=Q^{(j)}=e_{j}$ for every $j \geqslant 0$. It follows from (*) that $Q \in \mathcal{F}$. It also follows from $(*)$ that $Q$ is invertible, and $Q^{-1} \in \mathcal{F}$. Further, we can define "change of basis" map $A \in \mathfrak{M} \mapsto \tilde{A}=Q^{-1} A Q$. Since $Q, Q^{-1} \in \mathcal{F}$, this map is defined for every $A \in \mathfrak{M}$, one-to-one, onto, and $A=Q \tilde{A} Q^{-1}$. Clearly, $\tilde{A}$ describes the action of $A$ in terms of the $e_{i}$ 's. Finally, $A B=B A$ if and only if $\tilde{A} \tilde{B}=\tilde{B} \tilde{A}$ for $A, B \in \mathfrak{M}$.

Recall that $T$ denotes the Read operator introduced in the previous section. Since $T e_{j}=e_{j+1}$ for every $j \geqslant 0$, then $T Q f_{j}=Q f_{j+1}$, so that $Q^{-1} T Q f_{j}=f_{j+1}$ which implies $\tilde{T}=S$. Suppose $R T=T R$ for some $R \in \mathcal{B}\left(\ell_{1}\right)$, then $\tilde{R} S=S \tilde{R}$, so that $\tilde{R}=p(S)$ for some series $p(t)=\sum_{n=0}^{\infty} p_{n} t^{n}$ by Lemma 1 . Therefore, $R=Q \tilde{R} Q^{-1}=Q p(S) Q^{-1}=p\left(Q S Q^{-1}\right)=p(T)$. Since every bounded operator of the form $\sum_{n=0}^{\infty} p_{n} T^{n}$ commutes with $T$, we have proved the following proposition:

Proposition 2. The commutant of $T$ is the set of all bounded operators of the form $\sum_{n=0}^{\infty} p_{n} T^{n}$.

I would like to thank Prof. Y. A. Abramovich for suggesting this line of investigation to me and for our discussions. I am thankful to Professors C. D. Aliprantis, V. J. Lomonosov, and C. J. Read for their interest in this work.

We remark in conclusion that in [TV] we study the modulus $|T|$ of the Read operator of [R2], and we prove that, unlike $T$, the modulus $|T|$ does have an invariant subspace. 


\section{REFERENCES}

[L] V. I. Lomonosov, Invariant subspaces of the family of operators that commute with a completely continuous operator, Funktsional. Anal. i Prilozhen. 7 (1973), No. 3, 55-56. (Russian) MR 54:8319

[R1] C. J. Read, A short proof concerning the invariant subspace problem, J. Lond. Math. Soc., (2) 33 (1986), 335-348. MR 87m:47020

[R2] C. J. Read, Quasinilpotent Operators and the Invariant Subspace Problem, J.Lond.Math.Soc., (2) 56 (1997), No. 3, 595-606. MR 98m:47004

[TV] V. G. Troitsky, On the modulus of C. J. Read's operator, Positivity 2 (1998), No. 3, 257-264. CMP 99:04

Department of Mathematics, University of Illinois at Urbana-Champaign, 1409 West Green St., Urbana, Illinois 61801

E-mail address: vladimir@math.uiuc.edu 